\title{
The Design and Realization of a Rotary Inverted Pendulum Based on Efficient Control
}

\author{
Zhi-Yu ZHAO ${ }^{1, a}$, Gang DU ${ }^{1, b,{ }^{*},}$, Yun-Tao GOU ${ }^{1, c}$, Jun LI ${ }^{1,{ }^{d}}$ \\ ${ }^{1}$ School of Information Engineering China University of Geosciences, Beijing

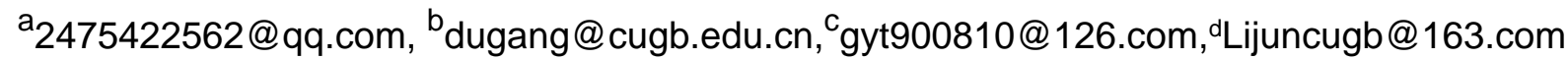 \\ ${ }^{*}$ Corresponding author
}

\begin{abstract}
Keywords: rotary inverted pendulum; efficient control; optimization; linear quadratic; fuzzy control; STM32.

Abstract: In this paper, a single-stage rotary inverted pendulum system is set up by using rotary encoder, servo motor and some mechanical structures. Lagrange equation is used to build up the mathematic model of single-stage rotary inverted pendulum. Linear quadratic regulator (LQR) and fuzzy control method are used to achieve the control functions of the rotary inverted pendulum. Experiments are taken to verify this control strategy through Matlab simulation. The system is realized on STM32 development board.
\end{abstract}

\section{Introduction}

Inverted pendulum is a naturally unstable system which has the characteristics of typical high-ordered, multivariable, non-linear and strong coupled. In recent years, the control methods of inverted pendulum have attracted widespread attentions.

The rotary inverted pendulum is developed from the linear inverted pendulum. The rod of pendulum is assembled on the arm which is driven by a servo motor to rotate in the horizontal plane and make the rod inverted. The rod can rotate in the vertical plane. The rotary inverted pendulum changes the linear inverted pendulum's horizontal control to rotary control. This kind of movement which makes the rod break away from the constraint of motion displacement also derives a new problem which is that circular motion comes along with non-linear centrifugal force which makes the whole system more complex and unstable.

However the most common used single-stage rotary inverted pendulum is tend to be highly integrated education suite which makes the requirement of control algorithm getting lower and lower, the anti-interference ability and the stability of the algorithm are going down. In this paper, based on the working principle of the inverted pendulum, we set up a single-stage rotary inverted pendulum system by using existing hardware resources, such as: rotary encoder, servo coding motor, servo motor driver and fixed support structure, etc. Compared with general integration of rotary inverted pendulum experimental box, our system has more noise and uncertainty, which means that there will be more interference to the stability of the swinging rod and that is why the requirements of our control algorithm is higher.

The organization of this paper is as follows. Section II presents the structure design of the rotary inverted pendulum system. Section III presents the mathematical modeling. Section IV presents the control methods. Section V provides the experimental results and Section VI concludes the paper.

\section{The Structure Design}

\section{The hardware design}

We choose STM32 development board as the control module. The ARM Cortex-M3 processor is an industry-leading 32 bits processor which is suitable for real-time applications with high certainly. STM32 is a high-performance platform with a low cost, and also a high-performance 32 bits CPU. We use the high speed characteristic and the flexible configuration of the ARM Cortex-M3 processor to control the rotary inverted pendulum. Additional uses of the LCD can display the location of the pendulum rod in real time. 
Faulhaber2342-L012 coreless motor with an encoder is chosen as the power module. Its voltage rating is $12 \mathrm{~V}$. It can realize the precise control through getting the position of the motor currently with the encoder. The Neurons intelligent motor driver module is chosen as the driver module. This module includes a L298N driver chip and a dsPIC33F MCU. Besides, it also has an access of the encoder. We can read the data from the encoder and transmit data to STM32 through serial communication. After the processing of the data, we can give the motor different input voltage to make it rotating in forward direction or reverse direction to make the pendulum rod in inverted state.

The sensing module of this system includes the rotary encoder of the pendulum rod and the encoder of the motor. It can get the increase or decrease of the angular displacement.

The rotary encoder is a detection device of angle, it can convert angular displacement into electric pulse or digital value through photoelectric principle.

We can figure out the rotation angle of both the arm and the pendulum rod. The principle of rotary encoder is shown as the figure below. We can judge its direction of rotation precesely by the phase relationships of chanel $\mathrm{A}$ and chanel $\mathrm{B}$. When the encoding disk rotates forward, the pulse waveform of chanel A is $\pi / 2$ earlier than B. Otherwise, the pulse waveform of chanel A is $\pi / 2$ later than B. We can also get the real-time velocity by processing the angle and time.

\section{Mathematical Modeling}

\section{Modeling}

The model of the rotary inverted pendulum is shown below. We establish $\mathrm{x}, \mathrm{y}, \mathrm{z}$ coordinates to describe the location of the arm and the rod. The arm rotates in the plane which is made up of $\mathrm{x}$ and $\mathrm{y}$ coordinates. $\theta_{1}$ is the angle between arm and $\mathrm{x}$. The rod rotates in the plane which is perpendicular to the arm. $\theta_{2}$ is the angle between rod and $\mathrm{z}$.

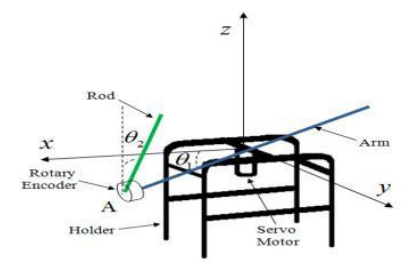

Figure 1. Rotary inverted pendulum reference coordinate system

The rotary inverted pendulum is generally composed of horizontal arm and homogeneous swinging rod which is fixed on the end of the arm. After ignoring air resistance and all sorts of friction, the rotary inverted pendulum can be abstracted as a rigid body system.

The coordinate system of the rotary inverted pendulum is set up as Fig.1.

The arm rotates around its midpoint. The rod rotates around the arm. According to the coordinate system we can get the coordinate of a particle on the arm. The length of the particle is dl, the distance between this particle and the midpoint of the arm is $1_{1}$.

$$
\left\{\begin{array}{l}
x=l_{1} \cos \theta_{1} \\
y=l_{1} \sin \theta_{1} \\
z=0
\end{array}\right.
$$

It is easy to know the kinetic energy of this particle is dT.

$$
d T=\frac{1}{2} \frac{m_{1}}{L} d l\left(\dot{x}^{2}+\dot{y}^{2}+\dot{z}^{2}\right)
$$

The kinetic energy of the arm $\mathrm{T}_{1}$ can be worked out by integral. 


$$
T_{1}=\int_{-L_{1}}^{L_{1}} d T=\frac{m_{1}}{2 \cdot 2 L_{1}} \int_{-L_{1}}^{L_{1}} l_{1}^{2} \dot{\theta}_{1}^{2} d l=\frac{1}{6} m_{1} L_{1}^{2} \dot{\theta}_{1}^{2}
$$

TABLE I. PARAMETERS

\begin{tabular}{|c|c|c|c|c|c|}
\hline Symbol & Description & Unit & Symbol & Description & Unit \\
\hline $\mathrm{m} 1$ & Mass of the arm & $\mathrm{kg}$ & $\mathrm{L}_{2}$ & $\begin{array}{l}\text { Length of the pendulum } \\
\text { rod }\end{array}$ & m \\
\hline $\mathrm{m} 2$ & Mass of the pendulum rod & $\mathrm{kg}$ & $1_{1}$ & $\begin{array}{l}\text { Distance between midpoint } \\
\text { and a particle on the arm }\end{array}$ & $\mathbf{m}$ \\
\hline $\mathrm{m} 3$ & $\begin{array}{l}\text { Mass of the rotary encoder } \\
\text { (include the fixture) }\end{array}$ & $\mathrm{kg}$ & $\mathbf{l}_{2}$ & $\begin{array}{l}\text { Distance between point } A \\
\text { and a particle on the rod }\end{array}$ & $\mathbf{m}$ \\
\hline $\mathrm{L}$ & Length of the arm & $\mathbf{m}$ & $\theta_{1}$ & Angle of the arm & degree \\
\hline $\mathbf{L}_{1}$ & $\begin{array}{l}\text { Distance between the rod and } \\
\text { the midpoint of the arm }\end{array}$ & m & $\theta_{2}$ & Angle of the pendulum rod & degree \\
\hline
\end{tabular}

We can also acquire the coordinate of a particle on the rod in the same way. The length of the particle is $\mathrm{dl}$, the distance between this particle and point $\mathrm{A}$ is $l_{2}$.

$$
\left\{\begin{array}{l}
x=L_{1} \cos \theta_{1}-l_{2} \sin \theta_{2} \sin \theta_{1} \\
y=L_{1} \sin \theta_{1}+l_{2} \sin \theta_{2} \cos \theta_{1} \\
z=l_{2} \cos \theta_{2}
\end{array}\right.
$$

The kinetic energy of this particle is dT.

$$
d T=\frac{1}{2} \frac{m_{2}}{L_{2}} d l\left(\dot{x}^{2}+\dot{y}^{2}+\dot{z}^{2}\right)
$$

The kinetic energy of the pendulum rod $\mathrm{T}_{2}$ can be worked out by integral.

$$
\begin{aligned}
& T_{2}=\int_{0}^{L_{2}} d T=\int_{0}^{L_{2}} \frac{1}{2} \times \frac{m_{2}}{L_{2}} d l\left(\dot{x}^{2}+\dot{y}^{2}+\dot{z}^{2}\right) \\
& =\left(\frac{1}{2} L_{1}^{2}+\frac{1}{6} L_{2}^{2} \sin ^{2} \theta_{2}\right) m_{2} \dot{\theta}_{1}^{2}+\frac{1}{2} m_{2} L_{1} L_{2} \\
& \cdot \cos \theta_{2} \dot{\theta}_{1} \dot{\theta}_{2}+\frac{1}{6} m_{2} L_{2}^{2} \dot{\theta}_{2}^{2}
\end{aligned}
$$

The rotary encoder and the fixture block can be regarded as a material point, its coordinate is in the equation below.

$$
\left\{\begin{array}{l}
x=L_{1} \cos \theta_{1} \\
y=L_{1} \sin \theta_{1} \\
z=0
\end{array}\right.
$$

The kinetic energy of this block is $\mathrm{T}_{3}$.

$$
T_{3}=\frac{1}{2} m_{3}\left(\dot{x}^{2}+\dot{y}^{2}+\dot{z}^{2}\right)=\frac{1}{2} m_{3} L_{1}^{2} \dot{\theta}_{1}^{2}
$$

The kinetic energy of this system can be got by summation operation. 


$$
\begin{aligned}
& T=T_{1}+T_{2}+T_{3} \\
& =\frac{1}{6} m_{1} L_{1}^{2} \dot{\theta}_{1}^{2}+\left(\frac{1}{2} L_{1}^{2}+\frac{1}{6} L_{2}^{2} \sin ^{2} \theta_{2}\right) m_{2} \dot{\theta}_{1}^{2} \\
& +\frac{1}{2} m_{2} L_{1} L_{2} \cos \theta_{2} \dot{\theta}_{1} \dot{\theta}_{2}+\frac{1}{6} m_{2} L_{2}{ }^{2} \dot{\theta}_{2}^{2}+\frac{1}{2} m_{3} L_{1}{ }^{2} \dot{\theta}_{1}^{2}
\end{aligned}
$$

We take the position of the rod's barycenter when in the original state as the zero potential energy point, and then the total potential energy of the system is V.

$$
\begin{aligned}
& V=m_{1} g \cdot \frac{1}{2} L_{2}+m_{2} g\left(\frac{1}{2} L_{2}+\frac{1}{2} L_{2} \cos \theta_{2}\right)+m_{3} g \cdot \frac{1}{2} L_{2} \\
& =\frac{1}{2} m_{1} g L_{2}+\frac{1}{2} m_{2} g\left(1+\cos \theta_{2}\right) L_{2}+\frac{1}{2} m_{3} g L_{2}
\end{aligned}
$$

The Lagrange dynamics equation can be written as the equation below.

$$
\frac{d}{d t}\left(\frac{\partial H}{\partial \dot{q}_{i}}\right)-\left(\frac{\partial H}{\partial \dot{q}_{i}}\right)=Q_{i} \quad i=1,2, \ldots, n
$$

Assuming that the generalized coordinate of the system is ${ }^{q_{i}}$, the generalized velocity is $\dot{q}_{i}, \mathrm{i}=1$, $2, \cdots, \mathrm{n} . Q_{i}$ is the generalized force corresponding to the generalized coordinates. In this paper, we use $q_{i}$ and $Q_{i}$ to express angle and torque.

When the system is a conservative system, the active force act on the system is a conservative force, which means that $Q_{i}=0$, and the Lagrange equation can be written in this form below:

$$
\frac{d}{d t}\left(\frac{\partial H}{\partial \dot{q}_{i}}\right)-\left(\frac{\partial H}{\partial \dot{q}_{i}}\right)=0
$$

$\mathrm{H}$ which is called as the Lagrange function in analytical mechanics is the difference of $\mathrm{T}$ (the kinetic energy of the system) and V(the potential energy).

By the formula above we can know the mathematical model of this system.

$$
\left[\begin{array}{c}
\dot{\theta}_{1} \\
\dot{\theta}_{2} \\
\ddot{\theta}_{1} \\
\ddot{\theta}_{2}
\end{array}\right]=\left[\begin{array}{cccc}
0 & 0 & 1 & 0 \\
0 & 0 & 0 & 1 \\
0 & -9.8486 & -13.4094 & 0.2037 \\
0 & 97.8257 & 25.0066 & -2.0234
\end{array}\right]\left[\begin{array}{c}
\theta_{1} \\
\theta_{2} \\
\dot{\theta}_{1} \\
\dot{\theta}_{2}
\end{array}\right]+\left[\begin{array}{c}
0 \\
0 \\
12.8987 \\
-24.0544
\end{array}\right] u
$$

\section{Control Methods}

\section{Linear quadratic optimal control method}

If the system is linear, the performance indicator is a quadratic function of state variables and control variables. This kind of dynamic system's optimization problem is called linear quadratic problem (LQR) .

Linear quadratic optimal control method is an important tool in designing feedback system. The system can stay near the equilibrium position by using LQR control method if the system is disturbed and deviate from the equilibrium position. And LQR can also make the dynamic error and the energy consumption optimally during the control process.

Linear quadratic regulator is aimed at linear time-invariant systems. 


$$
\left\{\begin{array}{l}
\dot{X}=A X+B U \\
Y=C X+D U
\end{array}\right.
$$

By determining the optimal control matrix, the performance indicators $\mathbf{J}$ of the system can be minimum.

$$
J=\frac{1}{2} \int_{0}^{\infty}\left(X^{T} Q X+U^{T} R U\right) d t
$$

In the formula above, the matrix $\mathrm{Q}$ and matrix $\mathrm{R}$ are used to equilibrate the weight of the state vector and the input vector. Matrix $\mathrm{Q}$ is a positive semi-definite matrix, matrix $\mathrm{R}$ is a positive definite matrix.

According to the extremum principle, we can reach the optimal control law.

$$
u^{*}(t)=-R^{-1} B^{T} P x(t)=-K x(t)
$$

In this formula, $\mathrm{K}$ is the optimal feedback gain matrix, and $\mathrm{P}$ is the constant positive definitematrix, both of which must satisfy the Riccati algebraic equation.

$$
A^{T} P+P A-P B R^{-1} B^{T} P+Q=0
$$

According to the Riccati algebraic equation, we can get the value of P. And the optimal feedback gain matrix $\mathrm{K}$ can be worked out too.

$$
K=R^{-1} B^{T} P
$$

The figure below is the diagram of LQR.

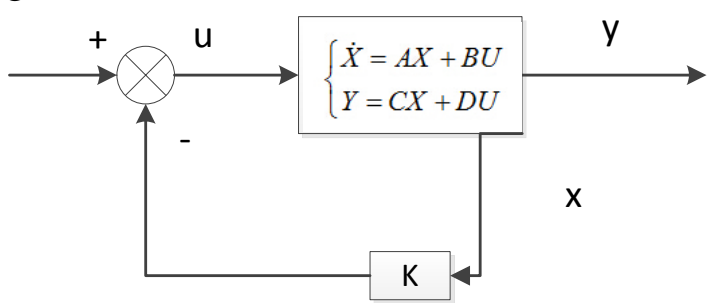

Figure 2. Diagram of LQR

\section{Fuzzy control method}

T-S fuzzy model is first proposed by the Japanese scholar Surgeon and Takagi and in 1985, which is a typical nonlinear fuzzy model of complex fuzzy dynamic model. The fuzzy rule it used is: the premise is classified based on whether there is a local linear relationship between system input and output, the conclusion can be expressed by polynomial linear equation so as to form various rules between the linear combination, which makes the global output of nonlinear systems possess the characteristic of good first describe.

$$
\begin{gathered}
R^{\mathrm{i}}: \text { if } x_{1} \text { is } A_{1}^{i} \text {, and } x_{2} \text { is } A_{2}^{i} \text {, and ...and } x_{k} \text { is } A_{k}^{i} \\
\text { then } y^{i}=p_{0}^{i}+p_{1}^{i} x_{1}+\cdots+p_{k}^{i} x_{k}
\end{gathered}
$$

If the fuzzy input vector is given $\left(x_{1}^{0}, x_{2}^{0}, \cdots, x_{m}^{0}\right)$, then the output of various rules is $y^{i}(i=1,2, \cdots, n)$, and through the weighted average we can get the output $\hat{y}$. 


$$
\hat{y}=\frac{\sum_{i=1}^{n} G^{i} y^{i}}{\sum_{i=1}^{n} G^{i}}
$$

In the formula above, $n$ is the number of fuzzy rule, ${ }^{y^{i}}$ can be calculated by the conclusion equation of the $i$-th rule, $G^{i}$ is the truth value of the $i$-th rule.

$$
G^{i}=\prod_{j=1}^{m} A_{j}^{i}\left(x_{j}^{0}\right)
$$

$\Pi$ is the fuzzy operator which usually takes the methods of minimizing operation or algebraic product operation.

The fuzzy controller is the core of the fuzzy control system. The performance of the fuzzy control system mainly depends on the structure of fuzzy controller, the fuzzy rules that are chosen, the compositional inference algorithm and the method of fuzzy decision, etc.

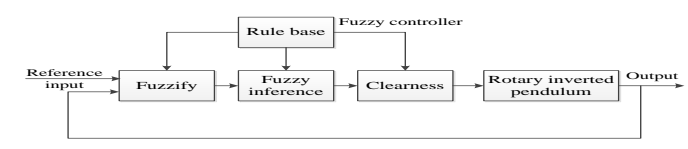

Figure 3. Fuzzy controller

Fuzzify is to transform the input parameter from accurate quantity to the formm that is required by the fuzzy controller. Through scale transform, the processed input conforms to the universe, the accurate quantity has been transfomed into fuzzy quantity after fuzzify. In this paper, we choose the gaussian fuzzifier as the fuzzification: $\mathrm{x} \in U$ is mapped to fuzzy set $A$ which is on $U$, this fuzzy set has gaussian type membership function.

$$
\mu_{A}(x)=\exp \left[-\left(\frac{x-x}{\sigma}\right)^{2}\right]
$$

The Fig. 4 is the input variable after gaussian type menbership function.

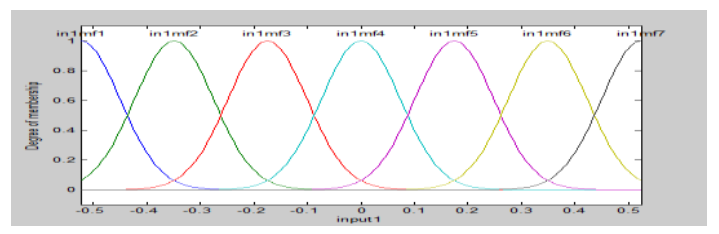

Figure 4. gaussian type membership function

The input variables are the angle of the pendulum rod and the angular velocity of the pendulum rod . We use negative big (NB), negative medium(NM), negative small(NS), zero(ZO), positive small(PS), positive medium(PM), positive big(PB) these seven lingual variables to describe the input variables. That is to say there are 49 kind of control rules, as the table below. 
TABLE II. RULE TABLE

\begin{tabular}{|l|l|l|l|l|l|l|l|}
\hline \multicolumn{1}{|c|}{$U(\mathrm{v})$} & -30 & -15 & -5 & 0 & 5 & 15 & 30 \\
\hline-45 & -11.77 & -6.27 & -3.13 & -1.55 & 0.02 & 3.09 & 8.34 \\
\hline-20 & -10.82 & -5.38 & -2.27 & -0.69 & 0.88 & 3.97 & 9.29 \\
\hline-5 & -10.24 & -4.85 & -1.75 & -0.17 & 1.40 & 4.50 & 9.86 \\
\hline 0 & -10.05 & -4.68 & -1.57 & 0 & 1.57 & 4.68 & 10.05 \\
\hline 5 & -9.86 & -4.50 & -1.40 & 0.17 & 1.75 & 4.85 & 10.24 \\
\hline 20 & -9.29 & -3.97 & -0.88 & 0.69 & 2.27 & 5.38 & 10.82 \\
\hline 45 & -8.34 & -3.09 & -0.02 & 1.55 & 3.13 & 6.27 & 11.77 \\
\hline
\end{tabular}

The LQR control law can only be ensured when the system is near the dynamic equilibrium point, so the LQR can only be used in fine control. For the situation when the system is deviate from the equilibrium point far away, the nonlinear factors of the system cannot be ignored. In this case, we use fuzzy control to do coarse control in a wider range. To make the inverted pendulum more stable, we designed the dual-controller structure.

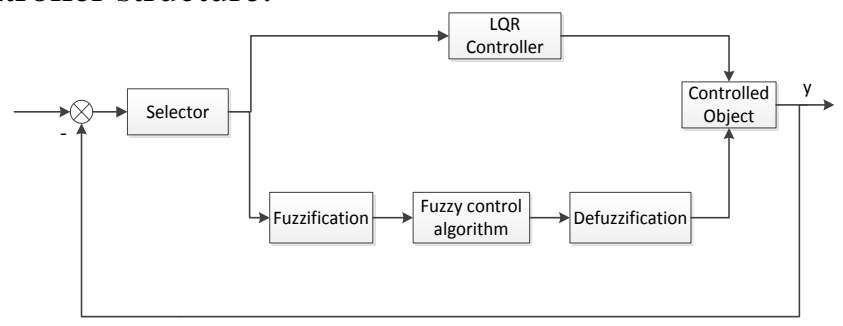

Figure 5. Dual-controller structure

\section{Function Realization and Simulation Test}

\section{The program design}

\section{A. The function realization}

Experiments indicate that the single-stage rotary inverted pendulum can initially realized swing up automatically, and keep in inverted state steadily. It can keep in stability state by conduct some stability adjustments when the disturbance is in a limited range.

The motor drives the arm do reciprocating motion when the rod is in the natural state, and let the rod reach inverted state. Then the system starts to run the stability control part to keep the rod in inverted state via control the direction and speed of the arm's velocity.

\section{B. The simulation test}

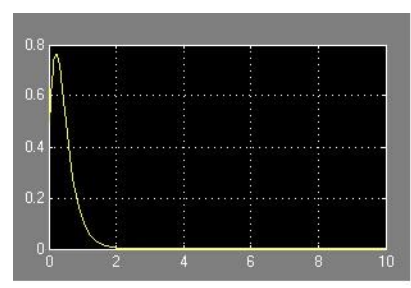

(a)

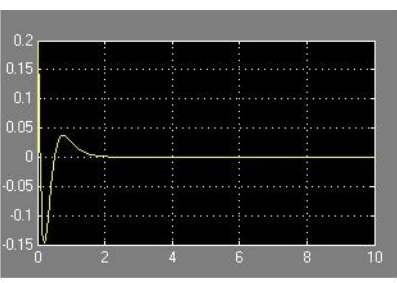

(b)

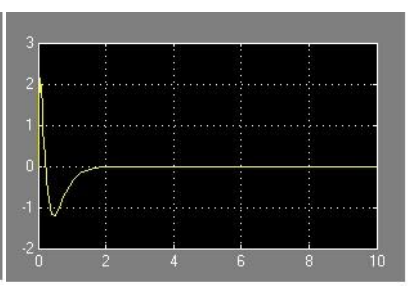

(c)

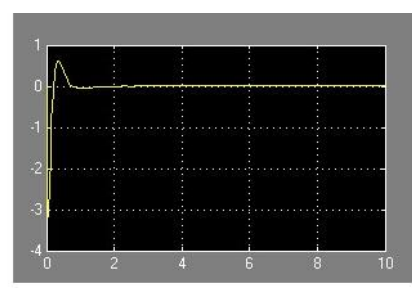

(d)

Figure 6. Graphs of $\theta_{1}, \theta_{2}, \dot{\theta}_{1}, \dot{\theta}_{2}$ after LQR control

As we can see in Fig.6, the four inputs enter into a stable state in a short time after the LQR control.

The values of the weighting matrix $\mathrm{Q}$ and $\mathrm{R}$ are very important in $\mathrm{LQR}$ control, the $\mathrm{Q}$ matrix is a symmetric matrix, $\mathrm{Q}_{11} \mathrm{Q}_{22}$ represents the weight of the angle of the arm and the rod. So $\mathrm{Q}_{11} \mathrm{Q}_{22}$ are chosen to be variables, and $\mathrm{Q}_{33}=\mathrm{Q}_{44}=0$. 


$$
Q=\left[\begin{array}{cccc}
Q_{11} & 0 & 0 & 0 \\
0 & Q_{22} & 0 & 0 \\
0 & 0 & Q_{33} & 0 \\
0 & 0 & 0 & Q_{44}
\end{array}\right]
$$

When the Q matrix takes different values, the control effect is different. Fig.7 is graph of the LQR simulation when the Q matrix takes different values. Fig.8 is graph of the LQR step response when the $\mathrm{Q}$ matrix takes different values. We can see that both the second $\mathrm{Q}\left(\mathrm{Q}_{11}=50, \mathrm{Q}_{22}=10\right)$ makes the control process shorter, and effect better.
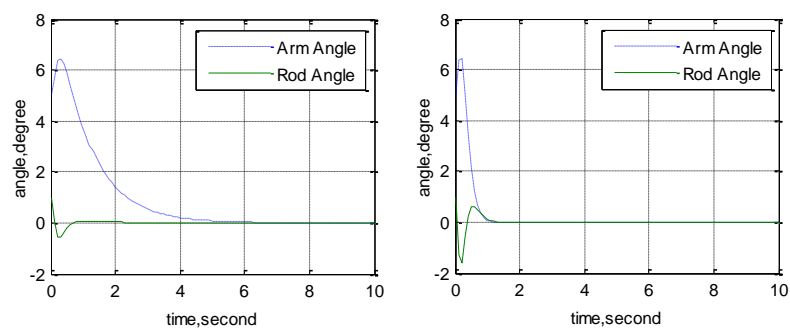

Figure 7. $\mathrm{LQR}$ simulation $\left(\mathrm{Q}_{11}=\mathrm{Q}_{22}=1\right),\left(\mathrm{Q}_{11}=50, \mathrm{Q}_{22}=10\right)$
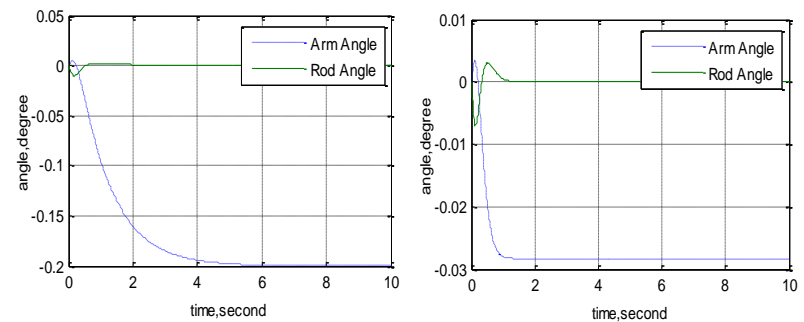

Figure 8. LQR step response $\left(\mathrm{Q}_{11}=\mathrm{Q}_{22}=1\right),\left(\mathrm{Q}_{11}=50, \mathrm{Q}_{22}=10\right)$

\section{Conclusion}

This paper has set up a model of single-stage rotary inverted pendulum system. The realization of this system is based on the STM32 development board. Photoelectric detection technology and control algorithm are used to realize swing up and the stability control of the rotary inverted pendulum. We strive to make the hardware circuit simple and give full play to the flexible feature of the software programming to meet the desired requirements.

The modeling of the rotary inverted pendulum is based on the Lagrange equation. We designed LQR control and fuzzy control method to realize the stability of the inverted pendulum.

Due to the limited experiment conditions, there're still something need to be improved. This will influence the reliability of the algorithm to some extent. In the future experiments, we will improve the equipments and also optimize the algorithm.

\section{References}

[1]ZHANG, S. and ZHU, S.A. (2004) Study on swing up control of rotary inverted pendulum. Journal of southern Yangtze university,10(3): 482-485.

[2]XIAO, L.L. and PENG, H. (2007) Upswing and stabilization control of a single inverted pendulum system based on the Lagrangian modeling Control Theory and Applications 1003-7241 (2007)04000405.

[3]WANG, Q.H. and WANG, D.G. (2013)Research on swing-up single circle inverted pendulum with error feedback.

[4]HOU, X.L. GU L.Z. and XU, X.H. (2003) Swing up control of a circular rail single inverted pendulum. Control and Decision, 18(4): 483-486. 
[5]HU, S.S. WANG, Z.Q. and HU, W.L. (2005) The optimal control theory and system.Beijing: Science Press, 2005.9.

[6]LIU, F. (2014) The design of single rotary inverted pendulum system based on fuzzy control. Science and Technology Innovation Herald 1674-098X(2014)02(a)-0083-01.

[7]LIU, H.L. and YANG, S. (2013) STM32 library develop practical guide. Beijing: China Machine Press, 2013.5.

[8]ZHANG, J. (2007) The application of MATLAB in control system.Beijing: Publishing House of Electronics Industry, 2007.5.

[9]Daniel, I.B. Juan ,S.C. and Luis, F. C. (2011)Rotary inverted pendulm with real time control, IEEE Latin American Robotics SymposiumLARS , DOI: 10.1109/LARC.2011.6086851. 